\title{
Twiss parameters and beam matrix formulation of generalized Courant-Snyder theory for coupled transverse beam dynamics
}

\author{
Moses Chung \\ Accelerator Physics Center, Fermi National Accelerator Laboratory, Batavia, IL 60510 \\ Hong Qin and Ronald C. Davidson \\ Plasma Physics Laboratory, Princeton University, Princeton, NJ 08543
}

(Dated: May 10, 2010)

\begin{abstract}
Courant-Snyder (CS) theory for one degree of freedom has recently been generalized by Qin and Davidson to the case of coupled transverse dynamics with two degrees of freedom. The generalized theory has four basic components of the original CS theory, i.e., the envelope equation, phase advance, transfer matrix, and the CS invariant, all of which have their counterparts in the original CS theory with remarkably similar expressions and physical meanings. In this Brief Communication, we further extend this remarkable similarity between the original and generalized CS theory, and construct the Twiss parameters and beam matrix in generalized forms for the case of a strong coupling system.
\end{abstract}


In recent papers [1, 2] Qin and Davidson generalized the Courant-Snyder theory [3-5] for one degree of freedom to the case of coupled transverse dynamics with two degrees of freedom using a time-dependent canonical transformation technique. Although there are several alternative parametrization methods for coupled transverse dynamics, such as the TengEdward parametrization [6-8], the Mais-Ripken parametrization [9-12], the normal-form method [13], and the Solution by LInear Matrices (SLIM) formalism [14], the Qin-Davidson parametrization is noteworthy in the sense that it retains the four basic components of the original Courant-Snyder theory, i.e., the envelope equation, phase advance, transfer matrix, and the Courant-Snyder invariant, with remarkably similar expressions and physical meanings to their counterparts in the original Courant-Snyder theory. This feature provides a formulation closer in structure to the original Courant-Snyder theory, and enables one to deal with more complicated coupled dynamics in the context of the well-established CourantSnyder formalism.

In this Brief Communication, we further investigate this remarkable similarity between the original and generalized Courant-Snyder theory, and construct the Twiss parameters $(\alpha, \beta$, and $\gamma)$ and the beam matrix $(\sigma)$ in generalized forms for the case of a strong coupling system. The generalized Twiss parameters define the shape and orientation of the $4 \mathrm{D} \mathrm{rms}$ hyper-ellipsoid which characterizes the equilibrium beam distribution in 4D phase space. Since all of the beam particles initially enclosed by the hyper-ellipsoid remain within that boundary along the transport channel (due to Liouville's theorem), we need only follow the evolution of the second moments of the beam distribution (i.e., the beam matrix), without tracking the trajectory of each individual beam particle [5]. Of course, other parametrization methods have their own particular generalizations of the Twiss parameters. However, none of them are explicitly derived from the envelope equation, which makes the extension of the original Courant-Snyder theory not as straightforward as the formulation described here.

The general form of the Hamiltonian for the coupled transverse dynamics is given by $[1,2]$

$$
H_{c}=\frac{1}{2} u^{T} A_{c} u,
$$

where

$$
A_{c}=\left(\begin{array}{cc}
\kappa(s) & R(s) \\
R^{T}(s) & I
\end{array}\right),
$$




$$
\kappa(s)=\left(\begin{array}{cc}
\kappa_{x} & \kappa_{x y} \\
\kappa_{x y} & \kappa_{y}
\end{array}\right),
$$

and $u=\left(x, y, p_{x}, p_{y}\right)^{T}$. Here, the $2 \times 2$ matrix $\kappa(s)$ is time-dependent and symmetric $\left(\kappa=\kappa^{T}\right), R(s)$ is an arbitrary, time-dependent $2 \times 2$ matrix, and $I$ is the $2 \times 2$ unit matrix. The variable $s$ is the path length that plays the role of a time-like variable. The superscript " $T$ " denotes the transpose operation of a matrix, and $p_{x}\left(p_{y}\right)$ is the scaled canonical momentum variable conjugate to the transverse coordinate $x(y)$ relative to the reference orbit. For a combination of all the linear components of a focusing lattice, i.e., the dipole, quadrupole, skew quadrupole, and solenoidal components, we obtain in the torsion-free curvilinear $(x, y, s)$-coordinate system [15]

$$
\begin{gathered}
\kappa(s)=\left(\begin{array}{cc}
\Omega^{2}+\kappa_{q}+\frac{1}{\rho_{x}^{2}} & \kappa_{s q}-\frac{1}{\rho_{x} \rho_{y}} \\
\kappa_{s q}-\frac{1}{\rho_{x} \rho_{y}} & \Omega^{2}-\kappa_{q}+\frac{1}{\rho_{y}^{2}}
\end{array}\right), \\
R(s)=\left(\begin{array}{cc}
0 & -\Omega \\
+\Omega & 0
\end{array}\right),
\end{gathered}
$$

where $\kappa_{q}$ is the quadrupole focusing coefficient, $\Omega$ is one-half of the normalized relativistic Larmor frequency associated with the solenoidal lattice [16], $\kappa_{s q}$ is the skew quadrupole coefficient, and $\rho_{x}\left(\rho_{y}\right)$ is the local bending radius in the $x(y)$-direction associated with the dipole field. Note that all of the elements in the matrices $\kappa(s)$ and $R(s)$ are generally time-dependent.

Applying the final results of the generalized Courant-Snyder theory obtained in Refs. $[1,2]$ to the Hamiltonian in Eq. (1), we express the solution for the transverse dynamics in terms of a time-dependent linear map from the initial condition $u_{0}$, i.e.,

$$
u(s)=M_{c} u_{0},
$$

where the transfer matrix $M_{c}$ is given by

$$
\begin{gathered}
M_{c}=Q^{-1} S^{-1} P^{-1} S_{0}, \\
Q^{-1}=\left(\begin{array}{cc}
Q_{4}^{T} & 0 \\
0 & Q_{4}^{T}
\end{array}\right), Q_{4}=\left(\begin{array}{cr}
\cos \theta & -\sin \theta \\
\sin \theta & \cos \theta
\end{array}\right), \\
S^{-1}=\left(\begin{array}{cc}
w^{T} & 0 \\
w^{-1} w^{\prime} w^{T} & w^{-1}
\end{array}\right),
\end{gathered}
$$




$$
\begin{aligned}
& P^{-1}=\left(\begin{array}{cc}
P_{1}^{T} & -P_{2}^{T} \\
P_{2}^{T} & P_{1}^{T}
\end{array}\right), \\
& S_{0}=\left(\begin{array}{cc}
\left(w_{0}^{-1}\right)^{T} & 0 \\
-w_{0}^{\prime} & w_{0}
\end{array}\right) .
\end{aligned}
$$

Here, $Q, S, P$, and $S_{0}$ are all symplectic matrices. Furthermore, $\theta^{\prime}=\Omega$ is the rate of the Larmor rotation phase, and $w=\left(\begin{array}{ll}w_{1} & w_{2} \\ w_{3} & w_{4}\end{array}\right)$ is the $2 \times 2$ envelope matrix satisfying the following non-commutative matrix envelope equation $[1,2]$

$$
w^{\prime \prime}+w \tilde{\kappa}=\left(w^{-1}\right)^{T} w^{-1}\left(w^{-1}\right)^{T},
$$

with $\tilde{\kappa}=Q_{4} \kappa Q_{4}^{-1}$, and $\left(w_{0}, w_{0}^{\prime}\right)$ denotes the initial conditions at $s=0$ for $w$ and $w^{\prime}$. The prime denotes a derivative with respect to $s$. The $4 \mathrm{D}$ rotation matrix $P^{-1}$ is determined from the generalized phase advance equations

$$
P_{1}^{\prime}=P_{2} \beta_{I}, P_{2}^{\prime}=-P_{1} \beta_{I},
$$

where $\beta_{I}=\left(w w^{T}\right)^{-1}$ is the matrix phase advance rate. The generalized Courant-Snyder invariant is

$$
I_{c}=u^{T} Q^{T} S^{T} S Q u,
$$

which is essentially the radius-squared of the $4 \mathrm{D}$ rotation in the normalized phase space coordinates $\bar{u}=S Q u$.

Now, we generalize the Twiss parameters by noting that, after a beam distribution reaches an equilibrium by phase-space filamentation, the contours of constant phase-space density become matched to the beam's single-particle trajectories [17]. Using the fact that $\tilde{\kappa}$ is symmetric, we rewrite the matrix envelope equation (12) in two parts according to

$$
\begin{gathered}
\left(w^{T} w\right)^{\prime \prime}+\tilde{\kappa}\left(w^{T} w\right)+\left(w^{T} w\right) \tilde{\kappa}=2\left[\left(w^{T} w\right)^{-1}+w^{T^{\prime}} w^{\prime}\right], \\
w^{\prime \prime} w^{T}=w w^{T^{\prime \prime}} .
\end{gathered}
$$

To obtain Eq. (15), we operate on Eq. (12) with $w^{T}(\cdots)+(\cdots)^{T} w$. Similarly, Eq. (16) is derived after operating on Eq. (12) with $(\cdots) w^{T}-w(\cdots)^{T}$. Due to the symmetric property of the matrix equations, Eq. (15) gives three independent coupled differential equations, whereas Eq. (16) gives only one. On the other hand, from the generalized form of the 
Courant-Snyder invariant in Eq. (14), we note that the beam particle is moving along the 4D hyper-ellipsoid, which is determined in the Larmor frame [16] by the matrix

$$
S^{T} S=\left(\begin{array}{cc}
\left(w^{T} w\right)^{-1}+w^{T^{\prime}} w^{\prime} & -w^{T^{\prime}} w \\
-w^{T} w^{\prime} & w^{T} w
\end{array}\right)
$$

Comparing Eqs. (15) and (17), we define the generalized Twiss parameters according to

$$
\begin{aligned}
& \alpha=-w^{T} w^{\prime}, \\
& \beta=w^{T} w \\
& \gamma=\left(w^{T} w\right)^{-1}+w^{T^{\prime}} w^{\prime} .
\end{aligned}
$$

Here, the generalized Twiss parameters $\alpha, \beta$, and $\gamma$ are $2 \times 2$ matrixes, and $\beta=\beta^{T}$ and $\gamma=\gamma^{T}$, while $\alpha \neq \alpha^{T}$ in general. The differential equation for the beta-function matrix $\beta$ becomes

$$
\beta^{\prime \prime}+\left[(\tilde{\kappa} \beta)+(\tilde{\kappa} \beta)^{T}\right]=2 \gamma
$$

and the derivative of $\beta$ yields

$$
\beta^{\prime}=w^{T^{\prime}} w+w^{T} w^{\prime}=-\left(\alpha+\alpha^{T}\right)
$$

both of which are non-commutative generalizations of their counterparts in the original Courant-Snyder theory with remarkably similar expressions. Here, we define $\beta=w^{T} w$, which is different from the definition in Refs. [1, 2], where $\beta$ was defined as the inverse of the matrix phase advance rate, i.e., $\beta=\beta_{I}^{-1}=w w^{T}$. Of course, for the uncoupled case, $w^{T} w=w w^{T}$.

Equation (16) provides very valuable additional information. Integration by parts of Eq. (16) yields

$$
w^{\prime} w^{T}-w w^{T^{\prime}}=\text { const. } \times\left(\begin{array}{cc}
0 & 1 \\
-1 & 0
\end{array}\right),
$$

where the integration constant is arbitrary, and can be determined from the initial conditions $\left(w_{0}, w_{0}^{\prime}\right)$. Since the time-dependent matrix $S$ in Eq. (9) should be symplectic, we require $S J S^{T}=J$ with $J=\left(\begin{array}{cc}0 & I \\ -I & 0\end{array}\right)$. This symplectic condition can be written explicitly as

$$
w^{\prime} w^{T}=w w^{T^{\prime}}
$$


Therefore, the initial conditions should be chosen in such a way that const. $=0$ in Eq. (23). For the uncoupled case, on the other hand, it always holds that $w^{\prime} w^{T}=w w^{T^{\prime}}$. It should be noted that Eq. (24) gives only one independent differential equation [see the explicit expression in Eq. (27)], and cannot replace the matrix envelope equation. The condition in Eq. (24) makes the expression for $S^{-1}$ much simpler, i.e.,

$$
S^{-1}=\left(\begin{array}{cc}
w^{T} & 0 \\
w^{-1} w^{\prime} w^{T} & w^{-1}
\end{array}\right)=\left(\begin{array}{cc}
w^{T} & 0 \\
w^{T^{\prime}} & w^{-1}
\end{array}\right),
$$

and readily gives the matrix version of the familiar relation between $\alpha, \beta$, and $\gamma$, i.e.,

$$
\beta \gamma=I+w^{T} w w^{T^{\prime}} w^{\prime}=I+\alpha^{2} .
$$

It should be emphasized here that, when solenoidal magnetic fields are present, the particle motion cannot be properly treated as a betatron motion (i.e., rotation around the reference orbit) in terms of the phase-space variables in the laboratory frame [18]. Strictly speaking, the above definitions of the Twiss parameters are meaningful only in the Larmor frame, in which the beam particles are indeed making the betatron motion.

So far, we have constructed the Twiss parameters in the context of the generalized Courant-Snyder theory. Once the matrix envelope equation (12) is solved, we can effectively describe the $4 \mathrm{D}$ hyper-ellipsoid on which the trajectories of the beam particles lie. To numerically integrate Eq. (12), we need to specify eight initial values, i.e., $\left(w_{1}, w_{2}, w_{3}, w_{4}\right)_{0}$ and $\left(w_{1}^{\prime}, w_{2}^{\prime}, w_{3}^{\prime}, w_{4}^{\prime}\right)_{0}$, which satisfy $\left(w^{\prime} w^{T}-w w^{T^{\prime}}\right)_{0}=0$. In terms of the elements of $w$, this condition can be expressed as

$$
\left(w_{1}^{\prime} w_{3}+w_{2}^{\prime} w_{4}-w_{3}^{\prime} w_{1}-w_{4}^{\prime} w_{2}\right)_{0}=0 .
$$

Therefore, we have indeed only seven unknown initial values. In a closed (or periodic) lattice system, it is desirable to find periodically matched solutions for $w$ to construct the Twiss parameters. The periodic matching conditions are

$$
\begin{aligned}
& \left(w_{1}, w_{2}, w_{3}, w_{4}\right)_{0}=\left(w_{1}, w_{2}, w_{3}, w_{4}\right)_{L} \\
& \left(w_{1}^{\prime}, w_{2}^{\prime}, w_{3}^{\prime}, w_{4}^{\prime}\right)_{0}=\left(w_{1}^{\prime}, w_{2}^{\prime}, w_{3}^{\prime}, w_{4}^{\prime}\right)_{L}
\end{aligned}
$$

where $L$ is the lattice periodicity length. When $w$ is the solution of the matrix envelope equation (12), it follows automatically from Eq. (23) that

$$
\begin{aligned}
& \left(w_{1}^{\prime} w_{3}+w_{2}^{\prime} w_{4}-w_{3}^{\prime} w_{1}-w_{4}^{\prime} w_{2}\right)_{0} \\
= & \left(w_{1}^{\prime} w_{3}+w_{2}^{\prime} w_{4}-w_{3}^{\prime} w_{1}-w_{4}^{\prime} w_{2}\right)_{L} .
\end{aligned}
$$


Hence, one of the eight constraints in Eqs. (28) and (29) is redundant, and only seven of them are indeed independent.

It is interesting to note that the matrix envelope equation (12) admits an orthogonal symmetry. Suppose that we have an arbitrary constant orthogonal matrix $C$, where $C^{T} C=$ I. Operating on Eq. (12) with $C(\cdots)$, and rearranging terms with $I=C^{T} C$, readily give

$$
\begin{aligned}
C w^{\prime \prime}+C w \tilde{\kappa} & =C\left(w^{-1}\right)^{T} w^{-1} C^{T} C\left(w^{-1}\right)^{T} \\
& =\left[(C w)^{-1}\right]^{T}(C w)^{-1}\left[(C w)^{-1}\right]^{T} .
\end{aligned}
$$

If $w$ is the solution of the matrix envelope equation (12), imposing the condition in Eq. (27) and the periodic boundary conditions in Eqs. (28) and (29), then it follows automatically from Eq. (31) that $\tilde{w}=C w$ is also a solution that satisfies $\left(\tilde{w}^{\prime} \tilde{w}^{T}-\tilde{w} \tilde{w}^{T^{\prime}}\right)_{0}=0$ and $\left(\tilde{w}, \tilde{w}^{\prime}\right)_{0}=\left(\tilde{w}, \tilde{w}^{\prime}\right)_{L}$. Indeed, this multiplicity of solutions is found in the original CourantSnyder theory as well. For example, the sign of the envelope function $w$ is not determined from the $1 \mathrm{D}$ envelope equation, but only the positive solution is used in calculations, for convenience [5]. On the other hand, it should be emphasized that the matrix $\beta=w^{T} w=$ $w^{T} C^{T} C w=\tilde{w}^{T} \tilde{w}$ is unique for all the solutions of $w$ in the same orthogonal group. In the similar manner, we can readily show that the matched solutions for $\alpha$ and $\gamma$ are also unique.

To describe the beam distribution in the 4D phase space, we now consider a multivariate Gaussian in the following form

$$
f(u)=\frac{1}{(2 \pi)^{2} \sqrt{\operatorname{det}(\sigma)}} \exp \left[-\frac{1}{2} u^{T} \sigma^{-1} u\right],
$$

where $\sigma=\left\langle u u^{T}\right\rangle$ is the beam matrix, and $\langle\cdots\rangle$ indicates the statistical average over the beam distribution. For simplicity, we assume $\langle u\rangle=0$, i.e., any centroid offset is neglected, or the coordinates are redefined with respect to the offset [19]. When an equilibrium state is reached in the $4 \mathrm{D}$ phase space, we can further assume that the contours of constant phasespace density are determined by a single invariant $I_{c}$. Of course, the general linear coupled motion is characterized by two invariants in each eigenmode, and thus, two mode emittances (not necessarily equal) are often used to describe an arbitrary beam distribution in 4D phase space. For a strong coupling system, however, there are rapid changes in the orientation of the eigenplanes themselves, in addition to the phase advance of the beam particles on each eigenplane. In this case, it is a natural approximation to assume equipartitioning of energy between the two degrees of freedom after many lattice periods, because of the phase-space 
filamentation (across the eigenplanes) associated with the nonlinear coupling terms [20], nonlinear space charge effects [21], and some stochastic processes [22]. For this equilibrium beam distribution, further emittance growth will be minimized, and the phase-space volume occupied by the beam particles is scaled with a single emittance without changing the shape and orientation of the hyper-ellipsoid. The rms hyper-ellipsoid is then determined by the $\exp [-1 / 2]$ contour of the Gaussian distribution function $f(u)$ [23]. By setting $I_{c}$ equal to the transverse rms emittance $\epsilon_{\perp}$ for the rms hyper-ellipsoid (which makes $\epsilon_{\perp}^{2}=\sqrt{\operatorname{det}(\sigma)}$ as in the usual convention), we find

$$
1=u^{T} \sigma^{-1} u=\frac{1}{\epsilon_{\perp}} u^{T} Q^{T}\left(\begin{array}{cc}
\gamma & \alpha^{T} \\
\alpha & \beta
\end{array}\right) Q u,
$$

and obtain the following expression for the beam matrix

$$
\sigma=\epsilon_{\perp} Q^{T}\left(\begin{array}{cc}
\gamma & \alpha^{T} \\
\alpha & \beta
\end{array}\right)^{-1} Q .
$$

If Eq. (25) is applied, we find

$$
\left(\begin{array}{cc}
\gamma & \alpha^{T} \\
\alpha & \beta
\end{array}\right)^{-1}=\left(\begin{array}{cc}
\beta & -\alpha \\
-\alpha^{T} & \gamma
\end{array}\right),
$$

and thus obtain a remarkably similar expression for the beam matrix as in the the original Courant-Snyder theory. Note that Eq. (34) is valid because $w^{\prime} w^{T}=w w^{T^{\prime}}$. We can readily show that $\sqrt{\operatorname{det}(\sigma)}=\epsilon_{\perp}^{2}$, as expected, and the volume enclosed by a $4 \mathrm{D}$ rms hyper-ellipsoid is $V_{4 \mathrm{D}}=\left(\pi^{2} / 2\right) \sqrt{\operatorname{det}(\sigma)}=\left(\pi^{2} / 2\right) \epsilon_{\perp}^{2}$, and the so-called trace invariant [18] is calculated to be $I_{2}=-\frac{1}{2} \operatorname{tr}(J \sigma J \sigma)=2 \epsilon_{\perp}^{2}$. We also note that $I_{c}=\epsilon_{\perp}$ only for the rms hyper-ellipsoid, while the average of the generalized Courant-Snyder invariant over the entire beam distribution is $\left\langle I_{c}\right\rangle=\int_{0}^{\infty} d I_{c}\left(I_{c} / 2 \epsilon_{\perp}\right)^{2} \exp \left[-I_{c} / 2 \epsilon_{\perp}\right]=4 \epsilon_{\perp}$. Finally, we assemble all of the calculations together into the following explicit form:

$$
\begin{aligned}
\sigma & =\left(\begin{array}{cccc}
\left\langle x^{2}\right\rangle & \langle x y\rangle & \left\langle x p_{x}\right\rangle & \left\langle x p_{y}\right\rangle \\
\langle y x\rangle & \left\langle y^{2}\right\rangle & \left\langle y p_{x}\right\rangle & \left\langle y p_{y}\right\rangle \\
\left\langle p_{x} x\right\rangle & \left\langle p_{x} y\right\rangle & \left\langle p_{x}^{2}\right\rangle & \left\langle p_{x} p_{y}\right\rangle \\
\left\langle p_{y} x\right\rangle & \left\langle p_{y} y\right\rangle & \left\langle p_{y} p_{x}\right\rangle & \left\langle p_{y}^{2}\right\rangle
\end{array}\right) \\
& =\epsilon_{\perp}\left(\begin{array}{cc}
Q_{4}^{T} & 0 \\
0 & Q_{4}^{T}
\end{array}\right)\left(\begin{array}{cc}
\beta & -\alpha \\
-\alpha^{T} & \gamma
\end{array}\right)\left(\begin{array}{cc}
Q_{4} & 0 \\
0 & Q_{4}
\end{array}\right) .
\end{aligned}
$$


Here, we note that $\sigma=\sigma^{T}$, and the beam matrix at the initial position $\sigma_{0}$ is related to $\sigma$ by $\sigma=M_{c} \sigma_{0} M_{c}^{T}$. For experimental measurements [19], the beam matrix is often expressed by the trace-space (geometrical) variables $X=\left(x, y, x^{\prime}, y^{\prime}\right)^{T}$ as

$$
\Sigma=\left\langle X X^{T}\right\rangle=U^{-1} \sigma\left(U^{T}\right)^{-1},
$$

with $u=U X$ and $U(s)=\left(\begin{array}{cc}I & 0 \\ R & I\end{array}\right)$. Note that the transformation $U$ is not symplectic.

The $(x, y)$-plane is the most obvious projection which shows the beam cross section under the influence of the coupling $[15,24]$. In general, the beam cross section becomes tilted due to the coupling, and the tilt angle varies along the beam transport channel. It can be readily shown that the $4 \mathrm{D}$ rms hyper-ellipsoid is projected onto a tilted ellipse given by

$$
\left(Q_{4} u\right)^{T} \beta^{-1}\left(Q_{4} u\right)=\epsilon_{\perp}
$$

Therefore, the physical meaning of the beta-function matrix $\beta$ is a description of the characteristic boundary of the tilted beam in the configuration space of the Larmor frame. Note that, in general, $\operatorname{det}(\beta)=[\operatorname{det}(w)]^{2} \neq$ const., which implies that the area of the beam cross section is not invariant. Similarly, the alpha-function matrix $\alpha$ describes not only the usual focusing and defocusing motions, but also the angular momentum induced by the coupling.

In this Brief Communication, by extending the generalized Courant-Snyder theory [1, 2], we have constructed the Twiss parameters and beam matrix in generalized forms for the case of a strong coupling system. The final expressions for the Twiss parameters $(\alpha, \beta$, and $\gamma)$ and the beam matrix $(\sigma)$ are remarkably similar to those of the original Courant-Snyder theory, and can therefore provide a compact and practical framework for the design and analysis of the beam transport channels with strong transverse coupling.

This research was supported by the U. S. Department of Energy.

[1] H. Qin and R. C. Davidson, Phys. Plasmas 16, 056701 (2009).

[2] H. Qin and R. C. Davidson, Phys. Rev. ST Accel. Beams 12, 064001 (2009).

[3] E. D. Courant and H. S. Snyder, Annals of Physics 3, 1 (1958).

[4] S. Y. Lee, Accelerator Physics (World Scientific, Singapore, 2004), Chapter 1.

[5] H. Wiedemann, Particle Accelerator Physics I (Springer, Berlin, 1999), Chapter 5. 
[6] L. C. Teng, Fermi National Accelerator Laboratory Report FN-229 (1971).

[7] D. A. Edwards and L. C. Teng, IEEE Trans. Nucl. Sci. NS-20, 885 (1973).

[8] D. Sagan and D. Rubin, Phys. Rev. ST Accel. Beams 2, 074001 (1999).

[9] G. Ripken, Deutsches Elektronen-Synchrotron Internal Report R1-70/04 (1970).

[10] I. Borchardt, E. Karantzoulis, H. Mais, and G. Ripken, Deutsches Elektronen-Synchrotron Internal Report 87-161 (1987).

[11] V. A. Lebedev and S. A. Bogacz, Jefferson Laboratory Technical Note 998 (2001).

[12] A. Wolski, Phys. Rev. ST Accel. Beams 9, 024001 (2006).

[13] A. J. Dragt, Handbook of Accelerator Physics and Engineering (World Scientific, Singapore, 1999), Chapter 2, Sec. 2.3.6.

[14] A. Chao, in Proceedings of 2008 European Particle Accelerator Conference, Genoa, Italy (2008), p. 2963.

[15] H. Wiedemann, Particle Accelerator Physics II (Springer, Berlin, 2003).

[16] R. C. Davidson and H. Qin, Physics of Intense Charged Particle Beams in High Energy Accelerators (World Scientific, Singapore, 2001).

[17] T. Wangler, RF Linear Accelerators (Wiley-VCH, Weinheim, 2008), 2nd ed.

[18] K.-J. Kim, Phys. Rev. ST Accel. Beams 6, 104002 (2003).

[19] M. G. Minty and F. Zimmermann, Measurement and Control of Charged Particle Beams (Springer, Berlin, 2003).

[20] E. Todesco and M. Giovannozzi, Phys. Rev. E 53, 4067 (1996).

[21] R. Kishek, P. O’Shea, and M. Reiser, Phys. Rev. Lett. 85, 4514 (2000).

[22] M. Reiser, Theory and Design of Charged Particle Beams (Wiley-VCH, Weinheim, 2008), 2nd ed., Chapter 6.

[23] J. B. Rosenzweig, Fundamentals of Beam Physics (Oxford University Press, Oxford, 2003).

[24] H. Qin, M. Chung, and R. C. Davidson, Phys. Rev. Lett. 103, 224802 (2009). 